\title{
IMPACT OF OBESITY ON SUSTAINED VIROLOGIC RESPONSE TO SOFOSBUVIR BASED REGIMENS IN THE EGYPTIAN CHRONIC HEPATITIS C PATIENTS
}

\section{By}

\author{
AHMED SAMIR ALLAM ${ }^{1 *}$, AYMAN GAMIL ANWAR ${ }^{1,}$ HAYTHAM MOHAMED \\ NASSER $^{2 \star \star}$, WALAA AHMED YOUSRY KABIEL ${ }^{3}$ AND AMIR M. FARID ${ }^{4}$ \\ Departments of Internal Medicine ${ }^{1}$, Radiology ${ }^{2}$, Clinical Pathology ${ }^{3}$ and Tropical \\ Medicine ${ }^{4}$, Faculty of Medicine, Ain Shams University, Cairo 11566, Egypt \\ ( ${ }^{\star}$ Correspondence: ahm82allam@gmail.com, ${ }^{* *}$ hmnh1980@gmail.com)

\section{Abstract}

Hepatitis $\mathrm{C}$ virus is one of the flaviviruses that can cause both acute and chronic hepatitis. Chronic HCV infection often follows a progressive course over years that could result in cirrhosis, hepatocellular carcinoma, and possible liver transplantation. Identifying modifiable risk factors that add to progression of the disease in HCV patients aids in choosing treatment approaches and overall disease management. Hepatic fibrosis may occur as a result of obesity, host, virus-mediated factors and hepatic steatosis. Nonetheless, the role of overweight and obesity on hepatitis C progression remains debatable. Nowadays, Direct Acting Antiviral therapy is the cornerstone of treatment of chronic hepatitis $\mathrm{C}$ virus infection aiming to eradicate HCV RNA, which is predicted by reaching sustained virologic response (SVR).

This prospective observational cross sectional study assessed the impact of obesity on the SVR to Sofosbuvir based therapy in patients with chronic HCV infection. The study included 188 chronic hepatitis $\mathrm{C}$ patients eligible for antiviral therapy according to the Egyptian guidelines issued by the National Committee for Control of Viral Hepatitis. G1: 93 obese patients with BMI $\geq 30 \mathrm{~kg} / \mathrm{m}^{2} \& \mathrm{G} 2: 95$ non obese patients with BMI $<30 \mathrm{~kg} / \mathrm{m}^{2}$. Each group was subdivided into 3 subgroups; GA received Sofosbuvir \& Daclatasvir, GB received Sofosbuvir \& Simeprevir while GC received Sofosbuvir \& Ledipasvir.

The results showed that overall SVR was $96.7 \%$ in G1 and $95.7 \%$ in G2. There was no association found between stage of fibrosis and SVR, and no significant association reached between obesity and sustained virologic response (SVR) to Sofosbuvir based regimens.

Keywords: Hepatitis C virus, Sofosbuvir, HCV/RNA, Sustained virologic response, Obesity.

\section{Introduction}

Egypt had the largest HCV epidemic worldwide as the prevalence of $\mathrm{HCV}$ reached $14.7 \%$. HCV killed an estimated 40 000 Egyptians a year and at least 1 of 10 of population aged 15 to 59 years was infected (WHO, 2014a).

The direct acting antiviral drugs were considered the cornerstone of HCV management. The goal of antiviral therapy is to eradicate HCV RNA, which is predicted by achieving sustained virologic response (SVR). Achieving SVR was associated with a 97 to $100 \%$ chance of being HCV/RNA negative during long-term follow-up in addition to decreasing liver-related deaths, hepatocellular carcinoma rates and liver-related complications, even among those patients with advanced liver fibrosis (Swain et al, 2010).
Several factors, both viral and host mediated have been shown to be responsible for the failure rate to antiviral treatment in the interferon-based era. Factors that were associated with worse response to interferonbased therapy were; male gender, old age, high body mass index (BMI), advanced liver fibrosis and high viral load. These factors had an important role in ma- nagement of decisions (David et al, 2018).

Obesity is defined as an abnormal or excessive fat accumulation that may impair health; Body mass index (BMI) the weight in kilograms divided by the square of the height in meters $\left(\mathrm{kg} / \mathrm{m}^{2}\right)$ is a commonly used index to classify overweight and obesity in adults. WHO defines overweight as a BMI $\geq 25$, and obesity as the BMI $\geq 30$ (WHO, 2014b). Some factors were postulated to cause a decreased response to the DAAs, 
which included hepatic steatosis, obesity complication, and the virus genotype (Conjeevaram et al, 2007).

The study aimed to assess the impact of obesity on the SVR to Sofosbuvir based therapy in patients with chronic $\mathrm{HCV}$ infection and to assess whether obesity decreases the efficacy of treatment and maintained SVR or not.

\section{Materials and Methods}

The study was a prospective observational cross sectional study. Subjects in this study included chronic hepatitis $\mathrm{C}$ patients eligible for antiviral therapy according to the Egyptian guidelines issued by the National Committee for Control of Viral Hepatitis (2016). They were recruited from the outpatient clinic of the Internal Medicine Department of Ain Shams University Hospitals, Cairo, Egypt. Informed written consent was obtained from each participant before enrolment in the study. The study was approved by the Research Ethics Committee of Ain Shams University, Number: 000017585.

All patients included fulfilled the following criteria with age $\geq 18$ years, chronic HCV with susceptibility to treatment criteria (non-cirrhotic naive patients with the following liver biochemical markers; total serum bilirubin $\leq 1.2 \mathrm{mg} / \mathrm{dl}$, serum albumin $\geq$ $3.5 \mathrm{~g} / \mathrm{dl}, \quad \mathrm{INR} \leq 1.2$ and Platelet count $\geq$ $150.000 / \mathrm{mm}^{3)}$ according to the protocol of Egyptian National Committee for Controlling HCV. While, patients whose age < 18 years old, had liver disease of non-HCV etiology, chronic HCV infection with difficult to treat criteria (cirrhotic patients, or with the following markers; albumin $<3.5 \mathrm{~g} / \mathrm{dl}$, total bilirubin $>1.2 \mathrm{mg} / \mathrm{dl}$, INR $>1.2$, and platelet count $<150,000 \mathrm{~mm} 3$ ), patients with HBV or HIV infection, patients with hepatocellular carcinoma, extra hepatic malignancy, renal disease; serum creatinine $>2.5 \mathrm{mg} /$ $\mathrm{dl}$, advanced severe illness were excluded.

At the start of study, 200 patients were eligible for treatment and meeting selection criteria, however at the end of the study ( 3 months after end of treatment), 12 patients were excluded [patients didn`t attend for follow up] and the remaining 188 patients completed the study.

Accordingly, patients who completed the study were divided into 2 groups: G1: 93 obese patients with BMI $\geq 30 \mathrm{~kg} / \mathrm{m}^{2}$ and $\mathrm{G} 2$ : 95 non obese patients with $\mathrm{BMI} \leq 30 \mathrm{~kg} / \mathrm{m}^{2}$.

The patients were again divided into 3 subgroups according to the received treatment in G1: GA (SOF/DAC): 35 patients received Sofosbuvir $(400 \mathrm{mg})$ plus Daclatasvir $(60 \mathrm{mg} /$ day) for 12 weeks, GB (SOF/ SIM): 27 patients received Sofosbuvir (400 $\mathrm{mg}$ ) plus Simeprevir (150mg/day) for 12 weeks and GC (SOF/LDV): 31 patients received Sofosbuvir (400mg) plus Ledipasvir (90mg) for 12 weeks. Also, 3 subgroups according to received treatment in G2: GA (SOF/DAC): 34 patients received Sofosbuvir (400mg) plus Daclatasvir (60mg/day) for 12 weeks, GB (SOF/SIM): 27 patients received Sofosbuvir (400mg) plus Simeprevir (150mg/ day) for 12 weeks and GC (SOF/LDV): 34 patients received Sofosbuvir (400mg) plus Ledipasvir (90mg) for 12 weeks.

They were subjected to the following: full history and physical examination with special emphasis on measurement of body mass index measurement $(\mathrm{BMI})=$ weight in kilograms divided by the square of height in meters $\left(\mathrm{kg} / \mathrm{m}^{2}\right)$. The following laboratory investigations were done: Complete blood count, liver function tests (albumin; ALT; AST; Total Bilirubin; direct bilirubin; prothrombin time and INR), Serum creatinine, fasting blood glucose \& HbA1c. Diagnosis of chronic hepatitis $\mathrm{C}$ infection was confirmed by: Positive HCV antibody using $3^{\text {rd }}$ generation immunoassay (enzyme immunoassay, EIA) \& detection of HCV/RNA, using quantitative PCR for HCV was assayed at 4, \&12 weeks (post-treatment end) and week 24 for assessment of sustained virologic response. Sustained virological response (SVR) was defined by undetectable serum HCV RNA by qualitative PCR assay (detection limit 15 IU/mL) 12 weeks after end of treatment. 
All patients were examined for other viral markers including HBsAg using enzyme immunoassays, HIV anti bodies using $3^{\text {rd }}$ generation ELISA. Serum AFP was quantitatively assessed using ELISA.

Abdominal ultrasound was done to detect sonographic signs of cirrhosis as coarse hepatic architecture, hypertrophy of the caudate lobe, atrophy of the right lobe, irregular borders, reduced liver size, ascites, splenomegaly, portal vein thrombosis, hepatic focal lesions. All were assessed for hepatic fibrosis by Fib4 score using Sterling's formula: Age (yr) x AST (IU/L) /platelet count $\left(10^{9} / \mathrm{L}\right) \times(\sqrt[2]{\operatorname{AST}}(U / L)$ after $($ WHO, 2016)

Statistical analysis: Data were coded, tabulated, and statistically analyzed using IBM SPSS statistics (Statistical Package for Social Sciences) software version 22.0, IBM Corp., Chicago, USA, 2013. Data were presented and suitable statistical analysis was done according to the type of data obtained for each parameter. The level of significance was taken at $\mathrm{P}$ value $(<0.001)$ is high significant, $\mathrm{P}$ value $(<0.005)$ was significant.

\section{Results}

The results were shown in tables $(1,2,3$, $4,5,6,7 \& 8$ )

Table 1: Comparison between G1 \& G2 regarding basal characteristics and ETR \& SVR.

\begin{tabular}{|c|c|c|c|c|}
\hline \multicolumn{2}{|c|}{ Variables } & G1 $(n=93)$ & G2 $(n=95)$ & $\mathrm{P}$ \\
\hline \multicolumn{2}{|c|}{ Age (years) } & $53.3 \pm 9.8$ & $51.4 \pm 10.7$ & $\wedge 0.206$ \\
\hline \multicolumn{2}{|c|}{ BMI $\left(\mathrm{kg} / \mathrm{m}^{2}\right)$} & $33.9 \pm 3.3$ & $25.3 \pm 3.2$ & $\wedge<0.001 *$ \\
\hline \multirow{2}{*}{ Sex } & Female & $29(31.1 \%)$ & $21(22.1 \%)$ & \multirow{2}{*}{ \#0.203 } \\
\hline & Male & $64(68.9 \%)$ & $74(79.9 \%)$ & \\
\hline \multirow{2}{*}{$\begin{array}{l}\text { Treatment } \\
\text { response }\end{array}$} & ETR & $90(96.7 \%)$ & $91(95.7 \%)$ & 20.497 \\
\hline & SVR & $90(96.7 \%)$ & $91(95.7 \%)$ & $a 0.497$ \\
\hline
\end{tabular}

Table 2: Comparison between G1 and G 2 regarding basal lab and FIB4

\begin{tabular}{|l|c|c|c|}
\hline \multicolumn{1}{|c|}{ Variables } & G1 & G2 & P \\
\hline HCV RNA $\left(x 10^{3} / \mathrm{mL}\right)$ & $510.9(145.8-1271.1)$ & $541.2(275.7-1170.3)$ & $\# 0.673$ \\
\hline AFP $(\mathrm{ng} / \mathrm{mL})$ & $7.2(4.2-11.6)$ & $4.5(2.4-9.4)$ & $\# 0.005^{*}$ \\
\hline Albumin $(\mathrm{gm} / \mathrm{dL})$ & $3.9 \pm 0.5$ & $3.9 \pm 0.5$ & $\wedge 0.764$ \\
\hline INR & $1.1 \pm 0.2$ & $1.1 \pm 0.2$ & ${ }^{\wedge} 0.465$ \\
\hline Creatinine $(\mathrm{mg} / \mathrm{dl})$ & $0.9 \pm 0.2$ & $0.9 \pm 0.2$ & ${ }^{\wedge} 0.183$ \\
\hline Hb $(\mathrm{gm} / \mathrm{dL})$ & $13.7 \pm 1.8$ & $13.9 \pm 1.4$ & ${ }^{\circ} 0.401$ \\
\hline Platelets $\left(\mathrm{x} 10^{3} / \mathrm{mL}\right)$ & $180.6 \pm 71.6$ & $182.9 \pm 62.8$ & $\wedge 0.808$ \\
\hline WBC $\left(\mathrm{x} 10^{3} / \mathrm{mL}\right)$ & $5.7 \pm 1.8$ & $6.0 \pm 1.8$ & $\wedge 0.278$ \\
\hline ALT $(\mathrm{IU} / \mathrm{L})$ & $52.8 \pm 32.2$ & $44.5 \pm 26.7$ & ${ }^{\wedge} 0.049^{*}$ \\
\hline AST $(\mathrm{IU} / \mathrm{L})$ & $52.7 \pm 32.1$ & $44.9 \pm 23.8$ & ${ }^{\wedge} 0.053$ \\
\hline Total bilirubin $(\mathrm{mg} / \mathrm{dL})$ & $0.9 \pm 0.5$ & $0.8 \pm 0.3$ & $\wedge 0.347$ \\
\hline FIB4 & $2.6 \pm 1.8$ & $2.3 \pm 1.6$ & $\wedge 0.167$ \\
\hline
\end{tabular}

^Independent t-test, \#Mann Whiney test, *Significant

There was significantly higher AFP \& G1 than G2, but without significant differALT and marginal significant higher AST in ence in both groups as regards FIB4

Table 3: Comparison between G1 \&G2 received SOF/DAC regimen regarding basal characteristics, ETR \& SVR.

\begin{tabular}{|c|c|c|c|}
\hline Variables & G1 $(n=35)$ & $\mathrm{G} 2(\mathrm{n}=34)$ & $\mathrm{P}$ \\
\hline Age (years) & $48.0 \pm 12.9$ & $47.5 \pm 13.0$ & $\wedge 0.866$ \\
\hline BMI $\left(\mathrm{kg} / \mathrm{m}^{2}\right)$ & $34.0 \pm 3.8$ & $24.3 \pm 3.3$ & $\wedge<0.001 *$ \\
\hline Female & $16(45.7 \%)$ & $12(35.2 \%)$ & \multirow[t]{2}{*}{$\# 0.220$} \\
\hline Male & $19(54.3 \%)$ & $22(64.8 \%)$ & \\
\hline HCV RNA (x10 $3 / \mathrm{mL})$ (Median (IQR) & $371.0(71.7-1460.0)$ & $330.3(65.3-940.0)$ & $\$ 0.967$ \\
\hline AFP (ng/mL) (Median (IQR) & $5.1(2.8-7.3)$ & $3.0(2.0-5.6)$ & $\S 0.033^{*}$ \\
\hline Albumin $(\mathrm{gm} / \mathrm{dL})$ & $4.0 \pm 0.5$ & $4.2 \pm 0.4$ & $\wedge 0.231$ \\
\hline INR & $1.1 \pm 0.1$ & $1.1 \pm 0.1$ & $\wedge 0.977$ \\
\hline ETR treatment response & $34(97 \%)$ & $33(97 \%)$ & $\not 0.964$ \\
\hline SVR treatment response & $34(97 \%)$ & $33(97 \%)$ & $a 0.964$ \\
\hline
\end{tabular}

^Independent t-test, §Mann Whitney test, \#Chi square test, ๙Fisher's Exat test, *Significant. 
No significant difference between $\mathrm{G} 1$ and $\mathrm{G} 2$ in SOD/DAC regimen regarding
ETR and SVR, But, G1 had significantly higher basal AFP.

Table 4: Comparison between G1 \&G2 received SOF/SIM regimen regarding basal characteristics, ETR\& SVR.

\begin{tabular}{|c|c|c|c|c|}
\hline \multicolumn{2}{|c|}{ Variables } & G1 (n=27) & $\mathrm{G} 2(\mathrm{n}=27)$ & $\mathrm{P}$ \\
\hline \multicolumn{2}{|l|}{ Age (years) } & $55.3 \pm 7.0$ & $54.1 \pm 9.4$ & $\wedge 0.539$ \\
\hline \multicolumn{2}{|l|}{ BMI $\left(\mathrm{kg} / \mathrm{m}^{2}\right)$} & $34.0 \pm 3.5$ & $24.3 \pm 2.8$ & $\wedge<0.001 *$ \\
\hline \multirow{2}{*}{ Sex } & Female & $10(37 \%)$ & $3(11.1 \%)$ & \multirow{2}{*}{ \#0.112 } \\
\hline & Male & $17(63 \%)$ & $24(88.9 \%)$ & \\
\hline \multicolumn{2}{|c|}{ HCV RNA (x103/mL) (Median (IQR) } & $309.0(85.6-942.8)$ & $641.1(325.3-2215.7)$ & $\S 0.022 *$ \\
\hline \multicolumn{2}{|c|}{$\mathrm{AFP}(\mathrm{ng} / \mathrm{mL})$} & $7.2(4.0-11.6)$ & $3.7(2.2-8.0)$ & $\S 0.028 *$ \\
\hline \multicolumn{2}{|c|}{ Albumin (gm/dL) } & $3.7 \pm 0.7$ & $3.7 \pm 0.5$ & $\wedge 0.709$ \\
\hline \multicolumn{2}{|c|}{ INR } & $1.2 \pm 0.2$ & $1.1 \pm 0.2$ & $\wedge 0.601$ \\
\hline \multirow{2}{*}{$\begin{array}{l}\text { Treatment re- } \\
\text { sponse }\end{array}$} & ETR & $25(92.5 \%)$ & $26(96.3 \%)$ & a0.978 \\
\hline & SVR & $25(92.5 \%)$ & $26(96.3 \%)$ & $a 0.978$ \\
\hline
\end{tabular}

No significant difference between groups received $\mathrm{SOF} / \mathrm{SIM}$ regimen as to
ETR \& SVR. G1 had significantly higher basal AFP and lower basal HCV RNA

\begin{tabular}{|c|c|c|c|}
\hline Variables & G1 $(n=31)$ & $\mathrm{G} 2(\mathrm{n}=34)$ & $\mathrm{P}$ \\
\hline Age (years) & $57.0 \pm 4.7$ & $52.9 \pm 7.7$ & $\wedge 0.056$ \\
\hline BMI $\left(\mathrm{kg} / \mathrm{m}^{2}\right)$ & $33.7 \pm 2.3$ & $27.6 \pm 2.1$ & $\wedge<0.001 *$ \\
\hline Female & $3(6.7 \%)$ & $6(17.6 \%)$ & \multirow[t]{2}{*}{$\mathrm{a} 0.424$} \\
\hline Male & $28(93.3 \%)$ & $28(82.4 \%)$ & \\
\hline HCV RNA (x10 $\left.{ }^{3} / \mathrm{ml}\right)$ (Median (IQR) & $837.9(411.0-1889.9)$ & $560.8(358.5-854.5)$ & $\S 0.033$ \\
\hline AFP (ng/mL) & $10.2(6.3-14.7)$ & $9.7(5.9-16.4)$ & $\S 0.912$ \\
\hline Albumin (gm/dL) & $3.9 \pm 0.3$ & $3.7 \pm 0.4$ & $\wedge 0.165$ \\
\hline INR & $1.2 \pm 0.1$ & $1.2 \pm 0.2$ & $\wedge 0.549$ \\
\hline Creatinine $(\mathrm{mg} / \mathrm{dL})$ & $0.9 \pm 0.1$ & $0.8 \pm 0.2$ & $\wedge 0.143$ \\
\hline ETR response & $31(100 \%)$ & $32(94.1 \%)$ & $\not a 0.982$ \\
\hline SVR response & $31(100 \%)$ & $32(94.1 \%)$ & $\not a 0.982$ \\
\hline
\end{tabular}

${ }^{\wedge}$ Independent t-test, §Mann Whitney test, aFisher's Exat test, *Significant.

No significant difference between groups received SOF/LDV regimen as to basal characters and ETR\& SVR Table 7: Comparison between G1 \&G2 received SOF/SIM regimen regarding CBC, liver functions and FIB4

\begin{tabular}{|c|c|c|c|c|}
\hline \multicolumn{2}{|c|}{ Variables } & G1 $(n=27)$ & G2 $(n=27)$ & ${ }^{\wedge} \mathrm{P}$ \\
\hline \multirow{4}{*}{$\mathrm{Hb}(\mathrm{gm} / \mathrm{dL})$} & Week-0 & $13.3 \pm 1.9$ & $13.8 \pm 1.6$ & 0.190 \\
\hline & Week-12 & $12.7 \pm 1.7$ & $13.0 \pm 2.2$ & 0.529 \\
\hline & Change (12-0) & $-0.6 \pm 1.5$ & $-0.8 \pm 2.7$ & 0.680 \\
\hline & \#P & $0.021^{*}$ & 0.072 & \\
\hline \multirow{4}{*}{ Platelets $\left(\times 10^{3} / \mathrm{mL}\right)$} & Week-0 & $170.7 \pm 52.6$ & $181.9 \pm 65.3$ & 0.431 \\
\hline & Week-12 & $175.5 \pm 54.4$ & $186.1 \pm 68.3$ & 0.477 \\
\hline & Change (12-0) & $0.6 \pm 61.7$ & $4.2 \pm 56.2$ & 0.799 \\
\hline & \#P & 0.334 & 0.282 & \\
\hline \multirow{4}{*}{$\begin{array}{c}\text { WBC } \\
\left(\times 10^{3} / \mathrm{mL}\right)\end{array}$} & Week-0 & $5.5 \pm 1.9$ & $5.2 \pm 1.9$ & 0.473 \\
\hline & Week-12 & $5.1 \pm 1.8$ & $5.2 \pm 2.0$ & 0.819 \\
\hline & Change (12-0) & $-0.4 \pm 1.8$ & $0.0 \pm 1.9$ & 0.427 \\
\hline & \#P & 0.275 & 0.977 & \\
\hline \multirow{4}{*}{$\begin{array}{c}\text { ALT } \\
(\mathrm{IU} / \mathrm{L})\end{array}$} & Week-0 & $51.3 \pm 39.2$ & $44.4 \pm 29.8$ & 0.408 \\
\hline & Week-12 & $22.3 \pm 10.5$ & $28.7 \pm 35.7$ & 0.312 \\
\hline & Change (12-0) & $-29.0 \pm 37.1$ & $-15.6 \pm 41.9$ & 0.163 \\
\hline & $\# \mathrm{P}$ & $<0.001^{*}$ & $0.037^{*}$ & \\
\hline \multirow{4}{*}{$\begin{array}{c}\text { AST } \\
\text { (IU/L) }\end{array}$} & Week-0 & $56.1 \pm 36.2$ & $46.5 \pm 27.1$ & 0.217 \\
\hline & Week-12 & $24.5 \pm 11.5$ & $24.4 \pm 7.8$ & 0.940 \\
\hline & Change (12-0) & $-31.7 \pm 35.1$ & $-11.9 \pm 49.6$ & 0.210 \\
\hline & \#P & $<0.001^{*}$ & $<0.001^{*}$ & \\
\hline \multirow{4}{*}{$\begin{array}{l}\text { Total bilirubin } \\
\quad(\mathrm{mg} / \mathrm{dL})\end{array}$} & Week-0 & $0.9 \pm 0.7$ & $0.8 \pm 0.4$ & 0.411 \\
\hline & Week-12 & $1.2 \pm 1.0$ & $1.3 \pm 1.2$ & 0.741 \\
\hline & Change (12-0) & $0.3 \pm 0.6$ & $0.4 \pm 1.1$ & 0.418 \\
\hline & \#P & $0.010^{*}$ & $0.028 *$ & \\
\hline \multirow{4}{*}{ FIB4 } & Week-0 & $2.9 \pm 1.9$ & $2.4 \pm 1.4$ & 0.195 \\
\hline & Week-12 & $2.0 \pm 1.6$ & $2.0 \pm 1.5$ & 0.975 \\
\hline & Change (12-0) & $-0.7 \pm 2.0$ & $-0.4 \pm 1.4$ & 0.545 \\
\hline & $\# \mathrm{P}$ & 0.074 & 0.086 & \\
\hline
\end{tabular}

\footnotetext{
${ }^{\wedge}$ Independent t-test, (Comparison between non obese \& obese), \#Paired T test (Comparison between times), *Significant.
} 
G1 high significant decrease in ALT \& AST, significant decrease in hemoglobin, significant increase in total bilirubin, G2 had high significant decrease in AST, significant decrease in ALT, significant increase in total bilirubin \& marginal significant decrease in hemoglobin. Groups had marginal significant decrease in FIB-4.

Table 6: Comparison between G1 \&G2 received SOF/DAC regimen regarding CBC, liver functions \& FIB4.

\begin{tabular}{|c|c|c|c|c|}
\hline \multicolumn{2}{|c|}{ Variables } & G1 $(n=35)$ & $\mathrm{G} 2(\mathrm{n}=34)$ & $\wedge \mathrm{P}$ \\
\hline \multirow{4}{*}{$\mathrm{Hb}(\mathrm{gm} / \mathrm{dL})$} & Week-0 & $14.0 \pm 1.4$ & $14.1 \pm 1.2$ & 0.713 \\
\hline & Week-12 & $13.0 \pm 1.2$ & $13.0 \pm 1.5$ & 0.902 \\
\hline & Change (12-0) & $-1.0 \pm 1.3$ & $-1.1 \pm 1.4$ & 0.802 \\
\hline & \#P & $<0.001^{*}$ & $<0.001^{*}$ & \\
\hline \multirow{4}{*}{$\begin{array}{c}\text { Platelets } \\
\left(\mathrm{x} 10^{3} / \mathrm{mL}\right)\end{array}$} & Week-0 & $236.1 \pm 78.2$ & $226.5 \pm 50.9$ & 0.542 \\
\hline & Week-12 & $223.7 \pm 71.0$ & $203.1 \pm 69.2$ & 0.231 \\
\hline & Change (12-0) & $-12.1 \pm 73.7$ & $-25.6 \pm 64.1$ & 0.422 \\
\hline & \#P & 0.347 & $0.026^{*}$ & \\
\hline \multirow{4}{*}{$\begin{array}{c}\text { WBC } \\
\left(x 10^{3} / \mathrm{mL}\right)\end{array}$} & Week-0 & $6.6 \pm 1.9$ & $6.8 \pm 1.6$ & 0.610 \\
\hline & Week-12 & $6.9 \pm 2.2$ & $6.0 \pm 1.9$ & 0.087 \\
\hline & Change (12-0) & $0.3 \pm 2.1$ & $-0.6 \pm 1.9$ & 0.070 \\
\hline & \#P & 0.474 & 0.062 & \\
\hline \multirow{4}{*}{$\begin{array}{c}\text { ALT } \\
(\mathrm{IU} / \mathrm{L})\end{array}$} & Week-0 & $42.5 \pm 24.3$ & $38.4 \pm 21.6$ & 0.460 \\
\hline & Week-12 & $22.0 \pm 7.6$ & $24.4 \pm 12.8$ & 0.350 \\
\hline & Change (12-0) & $-21.3 \pm 25.3$ & $-14.5 \pm 22.5$ & 0.246 \\
\hline & \#P & $<0.001^{*}$ & $<0.001 *$ & \\
\hline \multirow{4}{*}{$\begin{array}{l}\text { AST } \\
(\mathrm{IU} / \mathrm{L})\end{array}$} & Week-0 & $38.0 \pm 23.2$ & $36.2 \pm 17.8$ & 0.718 \\
\hline & Week-12 & $24.8 \pm 9.6$ & $23.2 \pm 8.9$ & 0.486 \\
\hline & Change (12-0) & $-13.7 \pm 23.9$ & $-13.0 \pm 15.8$ & 0.898 \\
\hline & \#P & $<0.001^{*}$ & $<0.001^{*}$ & \\
\hline \multirow{4}{*}{$\begin{array}{c}\text { Total } \\
\text { bilirubin } \\
\text { (mg/dL) }\end{array}$} & Week-0 & $0.8 \pm 0.3$ & $0.7 \pm 0.2$ & $0.030 *$ \\
\hline & Week-12 & $0.9 \pm 0.5$ & $0.7 \pm 0.3$ & $0.043^{*}$ \\
\hline & Change (12-0) & $0.0 \pm 0.4$ & $0.0 \pm 0.3$ & 0.550 \\
\hline & \#P & 0.645 & 0.703 & \\
\hline \multirow{4}{*}{ FIB4 } & Week-0 & $1.4 \pm 1.2$ & $1.3 \pm 0.7$ & 0.642 \\
\hline & Week-12 & $1.3 \pm 0.8$ & $1.2 \pm 0.6$ & 0.609 \\
\hline & Change (12-0) & $-0.1 \pm 1.2$ & $0.0 \pm 0.6$ & 0.789 \\
\hline & \#P & 0.604 & 0.655 & \\
\hline
\end{tabular}

^Independent t-test, (Comparison between non obese \& obese), \#Paired T test (Comparison between times), *Significant.

\section{Discussion}

The impact of obesity on SVR to antiviral therapy in CHC patients was heavily studied in the interferon- based era. Nevertheless, in the current era of highly effective direct acting antiviral drugs, estimation of factors that affect response to DAAs is still not fully studied. The high efficacy of these drugs will not only improve short and long term clinical outcome of HCV-related liver disease, but will likely impact on the incidence and prevalence of HCV-related extra hepatic diseases. (Ana et al, 2016).

In the present study, BMI in G1 was $33.9 \pm 3.3$ and $25.3 \pm 3.2$ in G2. The overall males were $64(68.9 \%)$ while females were $29(31.1 \%)$ in G1.In G2, males were 74
$(79.9 \%)$ and 21 female $(22.1 \%)$, without significant difference between groups regarding age, sex and treatment response. Also, there was no significant association between SVR and basal characteristics and labs as (viral load, age, sex, platelets, ALT and FIB4), as these factors did not have a significant effect on ETR and SVR. This agreed with Ana et al. (2016) who evaluated 363 CHC patients received Sofosbuvir based regimens as Sofosbuvir plus (Ledipasvir, Daclatasvir \& Simeprevir) with or without Ribavirin found that treatment failure roughly was $4 \%$, they estimated that outcome was not significantly influenced by viral load nor the treatment status, although, it was significantly influenced by advanced fibrosis. 
Table 8: Comparison between G1 \&G2 received SOF/LDV regimen regarding CBC, liver functions and FIB4

\begin{tabular}{|c|c|c|c|c|}
\hline \multicolumn{2}{|c|}{ Variables } & G1 $(n=31)$ & $\mathrm{G} 2(\mathrm{n}=34)$ & ${ }^{\wedge} \mathrm{P}$ \\
\hline \multirow{4}{*}{$\mathrm{Hb}(\mathrm{gm} / \mathrm{dL})$} & Week-0 & $14.2 \pm 0.9$ & $13.8 \pm 1.4$ & 0.129 \\
\hline & Week-12 & $13.1 \pm 0.6$ & $12.5 \pm 1.3$ & $0.030^{*}$ \\
\hline & Change (12-0) & $-1.1 \pm 1.0$ & $-1.2 \pm 1.2$ & 0.600 \\
\hline & \#P & $<0.001 *$ & $<0.001 *$ & \\
\hline \multirow{4}{*}{$\begin{array}{l}\text { P1stelets } \\
\left(\mathrm{x} 10^{3} / \mathrm{mL}\right)\end{array}$} & Week-0 & $127.3 \pm 16.9$ & $133.2 \pm 24.3$ & 0.279 \\
\hline & Week-12 & $164.2 \pm 11.7$ & $153.3 \pm 29.0$ & 0.063 \\
\hline & Change (12-0) & $36.5 \pm 20.7$ & $20.1 \pm 18.9$ & $0.002 *$ \\
\hline & \#P & $<0.001 *$ & $<0.001 *$ & \\
\hline \multirow{4}{*}{$\begin{array}{c}\text { WBC } \\
\left(x 10^{3} / \mathrm{mL}\right)\end{array}$} & Week-0 & $5.0 \pm 0.9$ & $6.1 \pm 1.6$ & $0.003^{*}$ \\
\hline & Week-12 & $5.0 \pm 0.9$ & $6.4 \pm 1.6$ & $<0.001 *$ \\
\hline & Change (12-0) & $0.1 \pm 1.0$ & $0.3 \pm 1.3$ & 0.494 \\
\hline & $\# \mathrm{P}$ & 0.516 & 0.180 & \\
\hline \multirow{4}{*}{$\begin{array}{c}\text { ALT } \\
\text { (IU/L) }\end{array}$} & Week-0 & $66.5 \pm 26.8$ & $51.7 \pm 27.4$ & $0.039 *$ \\
\hline & Week-12 & $27.4 \pm 7.3$ & $32.3 \pm 10.9$ & $0.044 *$ \\
\hline & Change (12-0) & $-39.1 \pm 25.9$ & $-19.4 \pm 27.4$ & $0.006^{*}$ \\
\hline & \#P & $<0.001 *$ & $<0.001 *$ & \\
\hline \multirow{4}{*}{$\begin{array}{c}\text { AST } \\
\text { (IU/L) }\end{array}$} & Week-0 & $65.8 \pm 29.9$ & $53.2 \pm 23.4$ & 0.072 \\
\hline & Week-12 & $27.6 \pm 6.5$ & $31.9 \pm 10.4$ & 0.057 \\
\hline & Change (12-0) & $-38.3 \pm 28.7$ & $-21.2 \pm 24.6$ & $0.017 *$ \\
\hline & \#P & $<0.001 *$ & $<0.001 *$ & \\
\hline \multirow{4}{*}{$\begin{array}{l}\text { Total bilirubin } \\
\quad(\mathrm{mg} / \mathrm{dL})\end{array}$} & Week-0 & $0.87 \pm 0.1$ & $0.99 \pm 0.3$ & $0.035^{*}$ \\
\hline & Week-12 & $0.94 \pm 0.1$ & $1.1 \pm 0.4$ & $0.042 *$ \\
\hline & Change (12-0) & $0.1 \pm 0.1$ & $0.1 \pm 0.3$ & 0.416 \\
\hline & \#P & $<0.001 *$ & $0.023^{*}$ & \\
\hline \multirow{4}{*}{ FIB4 } & Week-0 & $3.7 \pm 1.5$ & $3.3 \pm 1.8$ & 0.404 \\
\hline & Week-12 & $1.9 \pm 0.4$ & $2.1 \pm 1.2$ & 0.266 \\
\hline & Change (12-0) & $-1.9 \pm 1.3$ & $-1.2 \pm 1.6$ & 0.081 \\
\hline & \#P & $<0.001 *$ & $<0.001 *$ & \\
\hline
\end{tabular}

The 2014 Guidelines Development Group approved low- cost and effective methods for assessment of the degree of fibrosis. Liver biopsy, gold standard test for diagnosis, was not a suitable option due to its high cost, invasiveness and complications risk. Multiple non-invasive fibrosis tests based on blood indices and imaging modalities are now available as the aminotransferase/ platelet ratio index (APRI) and FIB-4 scores (WHO, 2016).

In the present study, the ultrasound and FIB4 score was used to diagnose liver fibrosis. Consequently, no association was found between the stages of fibrosis with SVR. This disagreed with Mangia et al. (2017) they evaluated predictors of sustained virological response 12 weeks after the end of treatment in hepatitis $\mathrm{C}$ virus patients in a trial that included $291 \mathrm{CHC}$ patients, 182 patients received SOF/DAC and 109 received SOF/RBV. SVR decreased from fibrosis stage $2(100 \%)$ to stage $3(91 \%)$ and 4 $(81.7 \%)(\mathrm{p}=0.05)$.
In the present study, no significant difference was found between both groups regarding basal FIB4 $\mathrm{p}$ value (0.167). This agreed with Leandro et al. (2006) who studied 3068 chronic hepatitis $\mathrm{C}$ patients and found that BMI was not identified as an independent factor for fibrosis. Also, it was with more than $80 \%$ of patients having $\mathrm{BMI} \geq 25$ $\mathrm{kg} / \mathrm{m}^{2}$, Verma et al. (2006) who found significantly higher rates of advanced fibrosis associated with older age, concurrent steatosis, and DM, but, without significant association was seen between obesity and advanced fibrosis.

However, Hu et al. (2009) evaluated a cohort of 460 consecutive HCV patients, reported significantly higher odds of advanced fibrosis among obese patients. Also, El Ray et al. (2013) in a study of 100 treatment naive chronic HCV patients found that significantly greater risk of advanced fibrosis associated with obesity. Harleen et al. (2015) analyzed twenty cohort studies found that obesity associated with increased risk of ad- 
vanced fibrosis in seven studies with effect size ranging from OR 1.08 to 7.69. The four studies did not report a significant association between obesity and advanced fibrosis.

In the present study, no significant association was found between obesity and sustaind virologic response (SVR) to the Sofosbuvir based regimens. Overall, SVR rates in the present study were $96.7 \%$ in G1 and 95.7\% in G2. More analysis revealed SVR in Sofosbuvir plus Daclatasvir regimen was $97 \%$ in both groups and in Sofosbuvir plus Simeprevir SVR was $92.5 \%$ in G1 and $96.3 \%$ in G2, without significance between them. But, in Sofosbuvir plus Ledipasvir, SVR was $100 \%$ in G1 \& $94.1 \%$ in $\mathrm{G} 2$, without significance between them. This disagreed with Lai et al. ( 2017) who found that patients with high BMI specially $\geq 40$ had a higher risk of treatment failure with LDV/SOF and that further studies are needed on pharmacokinetics of LDV/SOF in obese patients as well as consideration of this factor when selecting regimens for treatment of $\mathrm{HCV}$ patients. Tran et al. (2018) suggested that obesity, defined by BMI $\geq 30 \mathrm{~kg} / \mathrm{m}^{2}$, did not have a negative impact on virological response of CHC patients treated with DAA agents. The study was done on 168 patients treated with an oral DAA at a single center from 2015 to 2017. The following DAA regimens were prescribed: Sofosbuvir plus (Ledipasvir, Daclatasvir and Velpatasvir) \pm Ribavirin (RBV), Elbasvir/Grazoprevir and Paritaprevir/ Ritonavir-Ombitasvir and Dasabu vir \pm RBV. Accordingly, obesity did not impact sustained virologic response negatively in $\mathrm{CHC}$ patients receiving DAA therapy as noticed.

The limitations in this study were lack of determination of $\mathrm{HCV}$ genotype among the study cohort. But, HCV infection in Egypt is genotype 4. In fact, HCV genotype was a crucial predictor of SVR in the prior interferon era. Nonetheless, the current DAAs combinations are of high efficacy and pan genotypic coverage thus decreasing the role of $\mathrm{HCV}$ genotype in predicting treatment response. Also, the sample size was small.

\section{Conclusion}

The outcome results showed that obesity did not have effect on sustained virological response to Sofosbuvir based regimens in chronic hepatitis C Egyptian patients.

But, long-term prospective studies would confirm the link between obesity and hepatic steatosis and fibrosis to evaluate its effect on increase in BMI on HCV treatment.

\section{References}

Ana, A, Antonio, A, Vicente, S, et al, 2016: Rate and treatment predictors of treatment failure to all DAAs regimens outside clinical trials Antiviral therapy 10.3851/IMP3061.

Conjeevaram, HS, Kleiner, DE, Everhart, JE, et al, 2007: Race, insulin resistance and hepatic steatosis in chronic hepatitis C. Hepatology 45: 80-7.

David, L, Wyles, A, Thomas, L, et al, 2018: https://www.uptodate.com/contents/predictors-of -responseto-antiviral-therapy-for-chronic hepatitis-c-virus-infection?Topic 16500 Version 16.0.

El Ray, A, Asselah, T, Moucari, R, et al, 2013: Insulin resistance: a major factor associated with significant liver fibrosis in Egyptian patients with genotype 4 chronic hepatitis C. Eur. J. Gastroenterol. Hepatol. 25:421-7.

Harleen, K, Dyal, M, Aguilar, S, et al, 2015: Concurrent obesity, diabetes, and steatosis increase risk of advanced fibrosis among $\mathrm{HCV}$ patients: A Systematic Review. Dig. Dis. Sci. 60:2813-24.

Hu, SX, Kyulo, NL, Xia, VW, et al, 2009: Factors associated with hepatic fibrosis in patients with chronic hepatitis $\mathrm{C}$ : a retrospective study of a large cohort of US patients. J. Clin. Gastroenterol. 43:758-64.

Lai, MA, Witt, J, Ready, MP, Pauly, D, Witt, A, 2017: Obesity and high body weights predict ledipasvir/sofosbuvir treatment failure. J. Hepatol. 66:S516-9.

Leandro, G, Mangia, A, Hui, J, et al, 2006: HCV Meta-Analysis (on) Individual Patients' Data Study Group. Relationship between steatosis, inflammation, and fibrosis in chronic hepatitis C: a meta-analysis of individual patient data. Gastroenterology 130, 6:1636-42. Mangia, A, Santoro, R, Cenderello, G, et al, 2017: Predictors of sustained virological response 12 weeks 
after the end of treatment in hepatitis $\mathrm{C}$ virus GT3 infected patients receiving IFN-free antiviral treatments. J. Hepatol. 66:S95-132.

Swain MG, Lai MY, Shiffman ML, et al, 2010: A sustained virologic response is durable in patients with chronic hepatitis $\mathrm{C}$ treated with peginterferon alfa-2a and ribavirin. Gastroenterology 139:1593-9.

Tran, K, Kuwajima, VK, Tahan, AV, 2018: Impact of obesity on treatment of chronic hepatitis $C$ in interferon-free direct-acting antiviral era. Presented at AASLD, Liver Meeting. Study number 0681.

Verma, S, Bonacini, M, Govindarajan, S, et al, 2006: More advanced hepatic fibrosis in his- panics with chronic hepatitis $\mathrm{C}$ infection role of patient demographics, hepatic necroinflammation, and steatosis. Am. J. Gastroenterol. 101: 1817-23.

WHO, 2014a: Egypt steps up efforts against hepatitis C Retrieved from http://www. who. int/ features/2014/egypt-campaignhepatitisc/en

WHO, 2014b: Global Health Observatory (GHO) data overweight \& obesity. http:// www. who.int/gho/ncd/risk_factors/overweight/text/en. WHO, 2016: Guidelines for the screening, care, and treatment of persons with hepatitis $\mathrm{C}$ infection. April http://www.who.int/ hiv/topics/ hepatitis/en 